\title{
A Fully Reconfigurable Polarimetric Phased Array Antenna Testbed
}

\author{
Sudantha Perera, ${ }^{1}$ Yu Pan, ${ }^{1}$ Yan Zhang, ${ }^{1}$ Xining Yu, ${ }^{1}$ Dusan Zrnic, ${ }^{2}$ and Richard Doviak ${ }^{2}$ \\ ${ }^{1}$ Advanced Radar Research Center (ARRC) and Department of Electrical and Computer Engineering, University of Oklahoma, \\ Norman, OK, USA \\ ${ }^{2}$ National Severe Storms Laboratory (NSSL), National Oceanic and Atmospheric Administration (NOAA), Norman, OK, USA \\ Correspondence should be addressed to Yan Zhang; rockee@ou.edu
}

Received 7 January 2014; Accepted 26 May 2014; Published 6 August 2014

Academic Editor: Stefano Selleri

Copyright (C) 2014 Sudantha Perera et al. This is an open access article distributed under the Creative Commons Attribution License, which permits unrestricted use, distribution, and reproduction in any medium, provided the original work is properly cited.

\begin{abstract}
The configurable phased array demonstrator (CPAD) is a low-cost, reconfigurable, small-scale testbed for the dual-polarized array antenna and radar prototype. It is based on the concept that individual transmit and receive (TR) modules and radiating elements can be configured in different ways to study the impact of various array manifolds on radiation pattern performance. For example, CPAD is configured as (a) a $4 \times 4$ planar array, (b) a planar array with mirror configuration, and (c) a circular array to support the multifunctional phased array radar (MPAR) system risk reduction studies. System descriptions are given in detail, and measurements are made and results are analyzed.
\end{abstract}

\section{Introduction}

Risk mitigation of a large-scale, multifunctional phased array radar (MPAR) usually involves [1] designing, fabricating, and testing small-scale subarray panels and [2] scaling up to larger array systems. Often these subarrays are based on assumptions about the linear or planar array manifold. However, there have been other options for the array manifold, such as the cylindrical array [3] and the sparse array [4]. Although theoretical analysis has claimed unique advantages for each of the different array manifold options, testing the different design options with full-scale systems built for each option is expensive and time consuming. A laboratory scale testbed that can be instrumented for fair, quantitative, and precise design comparisons is needed.

Because the main focus of our investigation is how the array manifold configurations affect the polarimetric array radiation pattern, a configurable phased array demonstrator (CPAD) is developed based on integrating the radiating element with the individual T/R module. The array manifold, as well as the beamforming and control circuitry, can be rapidly reconfigured (Figure 1), as in the case of the planar subarray (Figure 2(a)) and the circular/conformal array (Figure 2(b)).
Multiple circular structures can be stacked together to analyze the performance of manifolds on scalable and complicated curvature surfaces. It is shown that a simple measurement procedure can be developed, and accurate measurement data for the scalable validation of candidate array antenna system designs can be provided with this concept.

This paper is organized as follows. In Sections 2 and 3 , basic system architecture and CPAD TR module designs are discussed. Section 4 introduces CPAD configuration for both planar and circular arrays. Sections 5 and 6 describe the measurement method and procedures, as well as detailed measurement results. Discussion and conclusions are found in Section 7.

\section{Instrumentation Components}

2.1. Front End. The individual TR modules of the CPAD have independent antennas and control signals so that they can easily fit into different manifold structures. Various antenna element designs can be attached to the TR modules as long as they are within the maximal allowable dimensions $\left(3^{\prime \prime} \times 3^{\prime \prime}\right.$ for the current configuration). With this architecture, the 


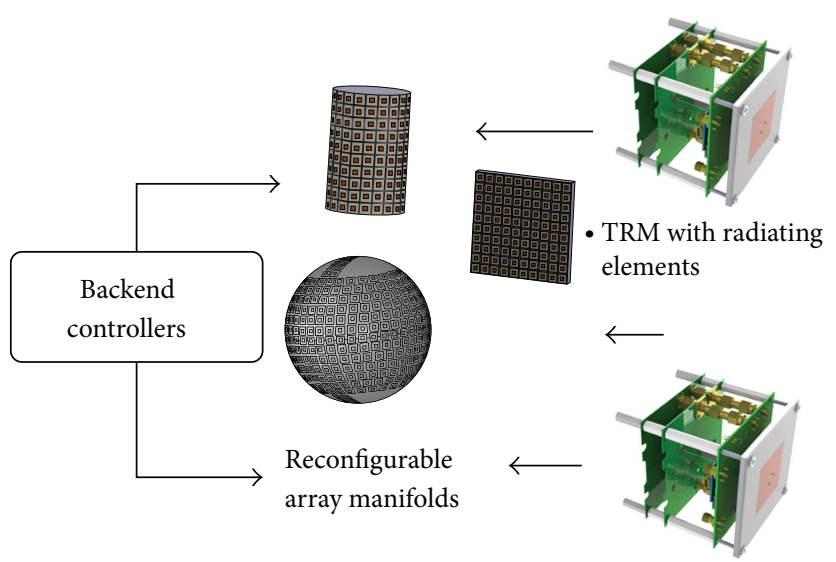

Figure 1: The CPAD concept.

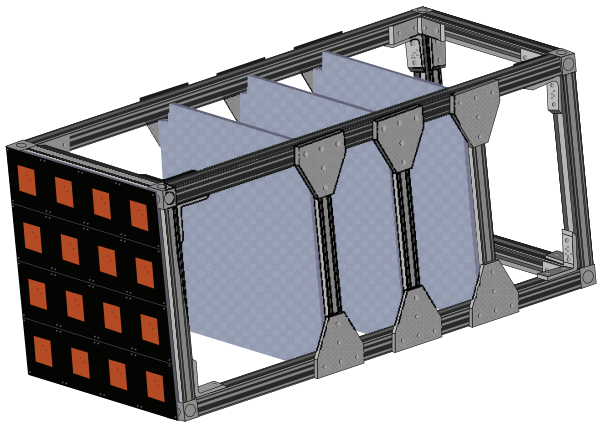

(a) An example of a polarimetric planar array configuration

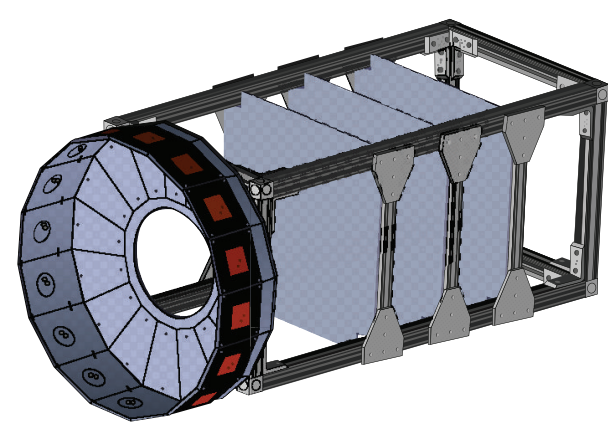

(b) An example of a polarimetric circular array configuration

FIgURE 2: 3D model of example CPAD configurations.

CPAD can function as a subarray in a large-scale array system, or it can be reconfigured to study the performance of different array manifold designs for the risk mitigation missions, including the cost and performance, of large systems. An initial demonstration of the CPAD is developed with 16 radiating elements $(4 \times 4)$, which are separated by $0.7 \lambda$ spacing. The operating frequency is $2.705 \mathrm{GHz}$. The front-end circuitry is shown in Figure 3.

2.2. TR Modules. An introduction of the development of the TR module was presented earlier in [5]. The structure of the CPAD TR module is depicted in Figure 3. The CPAD TR modules are designed to have all the required circuit elements in a typical modern phased array radar with self-contained features; that is, the individual antenna elements are attached directly to the TR modules, and the microcontroller unit (MCU) in each TR module can store its own program to control the phase shift and attenuation of each polarimetric channel. At the same time, a master MCU board can communicate with the MCUs within all the TR modules through a serial peripheral interface (SPI) for program loading, synchronizing, and so forth. TR modules have the same external global triggering/control signal, which can have a 5\% duty cycle without overloading the high power amplifier (HPA). This triggering signal is used to control the T/R switches which enable sharing the same phased shifter (PS) and attenuator (ATT) for both transmission and reception signals as in Figure 3. The amplifiers in TRMs (LNA, preamplifier, and HPA) can operate in both continuous wave (CW) and pulsed modes. In laboratory measurements, low-power continuous transmission is more appropriate because it avoids possible electromagnetic hazards for equipment and people, and it is simpler to implement. For field demonstrations, the higher power pulsed mode is necessary since high peak power is important for effective range performance. When operating in pulse mode, the amplifiers in all the TR modules are synchronized with the same triggering signal.

Each TR module consists of LNA, preamp, HPA, phase shifter, attenuator, and polarized microstrip patch radiating elements. The power divider/splitters in the CPAD are common components of the beamformer for both transmission and reception. The same architecture is used for both horizontal and vertical polarizations. Images of individual TRMs are shown in Figure 4. Each TR module contains four PCB 


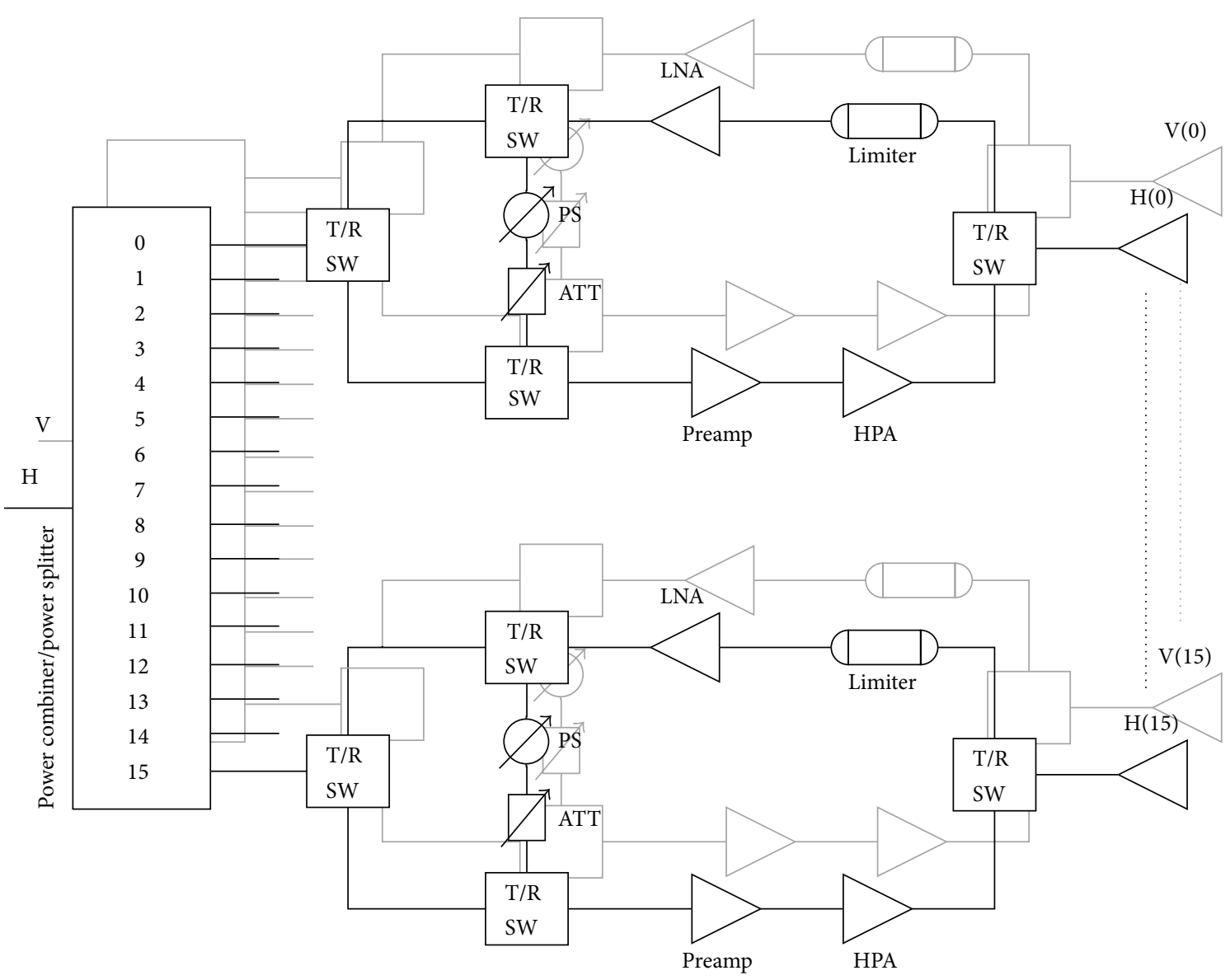

FIGURE 3: An example of CPAD front-end system architecture for planar array demo.

boards as well as the microstrip patch antenna. As shown in Figure 4(a), the RF transceiver and controller board have been assembled with TR switches, a phased shifter, LNA, attenuator, and limiter. The MCU board is attached to the back of the RF transceiver and controller board. For the high power version, the power amplifier boards for the $\mathrm{H}$ and $\mathrm{V}$ channels are identical. Figure 4(c) shows a GaN HPA board for one channel. For low-power HPA, only the preamplifiers are used, and the two channels are integrated on the same board. All the TRMs are carefully tested to ensure their operational stability before installing them into the array system.

\section{CPAD Integration}

3.1. Planar Array. An initial demonstration of the CPAD is via a simple planar subarray system, which is shown in Figure 5(a). 16 TR modules are mounted on the $30 \mathrm{~cm} \times$ $30 \mathrm{~cm}$ aluminum plate of the CPAD structure to construct a $4 \times 4$ polarimetric antenna array. In this demonstration, the radiation elements can be either arranged normally (all in the same orientation) or in a mirror configuration [6] (the orientation in Figure 12) if cross-polarization levels need to be suppressed at broadside. The array manifold in Figure 5(a) also shows three layers of electronics: (1) the power divider, combiner, and cabling, (2) the TR module and antennas, and (3) the master MCU for the subarray and power suppliers (110 V AC to $28 \mathrm{~V}$ DC converters) which are mounted on the third plate. At the subarray level, the master MCU can communicate with a computer through a USB port and transfer the phase/attenuation codes of all the TRMs.

A LabVIEW program runs on the computer with a simple graphic user interface (GUI). The antenna elements are divided into three regions, labeled as 1,2 , and 3 , on a $4 \times 4$ array (Table 1 ). The first region has four elements near the center. The second region has elements at the four corners. The third region has the remaining eight elements. By adjusting the relative power to elements in each of the regions, we can optimize the amplitude tapering. The user interface accepts specified phase shifts and attenuations to steer and form the beam.

3.2. Circular Array. The circular array (or ring array) has many applications in communication, radar, and directional finding systems [7]. It is also an important candidate for the polarimetric MPAR manifold [2]. The CPAD has been 


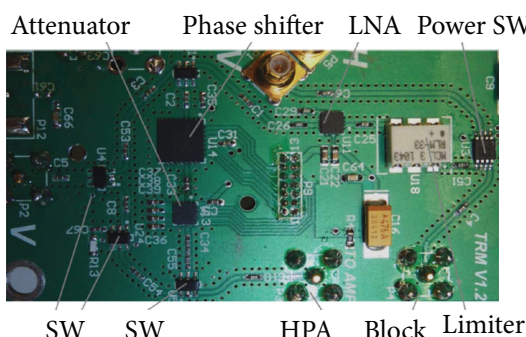

(a) A close look of V channel RF circuit

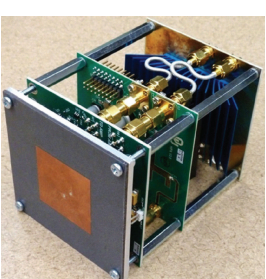

(b) A completed TR module with RF board, two $40 \mathrm{dBm}$ HPAs, Rf board, and a dualpolarized patch antenna

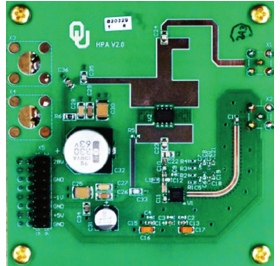

(c) The GaN HPA board. NPT25015 transistor from NITRONEX is used

FIgURE 4: TR module.

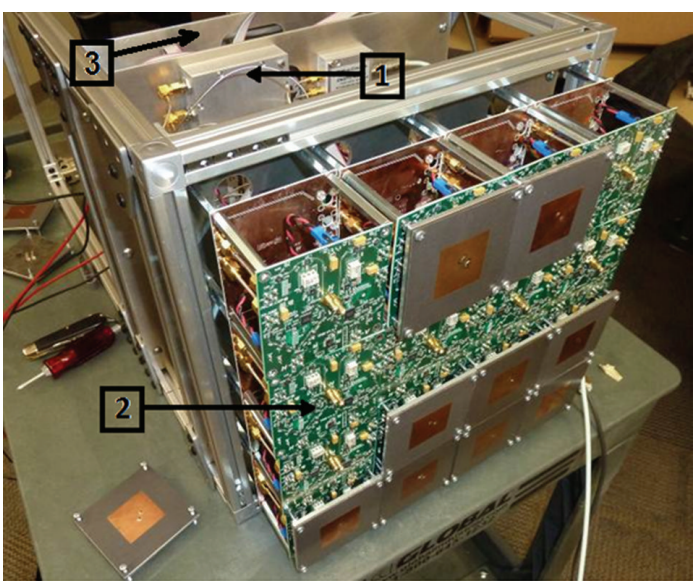

(a) Planar subarray manifold to study the polarimetric array pattern variation with different scanning angles. (1) is the beamformer, (2) is the RF board, and (3) is power supply

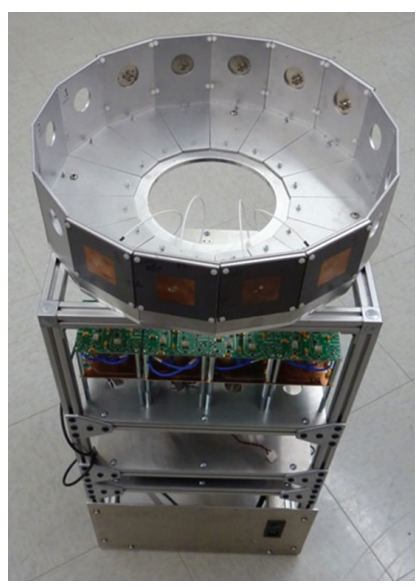

(b) 4-element circular array manifold to study the polarimetric array pattern variation with different scanning angles

Figure 5: The CPAD being integrated.

TABLE 1: Three regions of the antenna elements distribution on the $4 \times 4$ array.

\begin{tabular}{lcc}
\hline Region & Element labels (refer to Figure 8) & Attenuation \\
\hline 1 & $(1,1),(1,2),(2,1)$, and $(2,2)$ & $0 \mathrm{~dB}$ \\
2 & $(0,0),(0,3),(3,0)$, and $(3,3)$ & $15 \mathrm{~dB}$ \\
3 & $(0,1),(0,2),(1,0),(2,0)$, and $(3,1)$ & $5 \mathrm{~dB}$ \\
& $(3,2),(1,3)$, and $(2,3)$ & \\
\hline
\end{tabular}

configured as a 16-element circular array for the initial conformal array demonstration. During pattern measurements, the CPAD was configured as either a 4-element (depicted in Figure 5(b)) or an 8-element circular array. Integration of the circular array is identical to the planar CPAD except for the antenna manifold on top of the RF system.

\section{Measurement Method}

The initial measurements of the planar CPAD were made in an anechoic chamber at the University of Oklahoma using a vector network analyzer (VNA) (Agilent E8364B PNA network analyzer is used). The tests performed were for the receive-mode array and the low power transmit-mode array (the HPAs were not activated).

Antenna measurement setup is shown in Figure 6. The CPAD test antenna array was mounted on a positioning system consisting of an azimuth rotary stage (soloist singleaxis digital servo controller is used to control the azimuth 


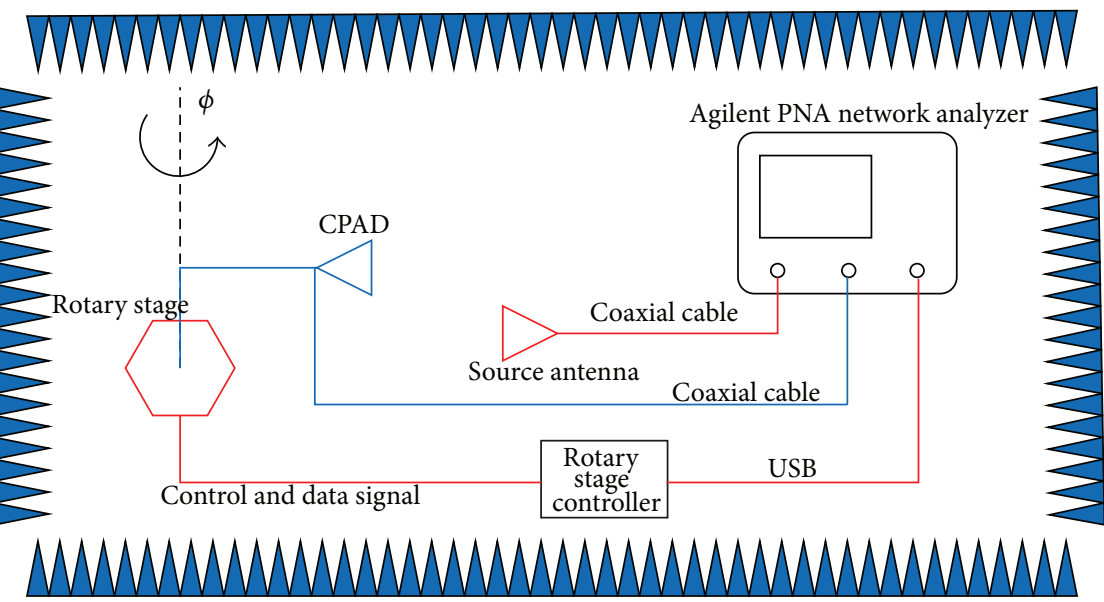

FIGURE 6: Block diagram of $\mathrm{H}-\mathrm{H}, \mathrm{H}-\mathrm{V}, \mathrm{V}-\mathrm{V}$, or $\mathrm{V}-\mathrm{H}$ pattern measurements.

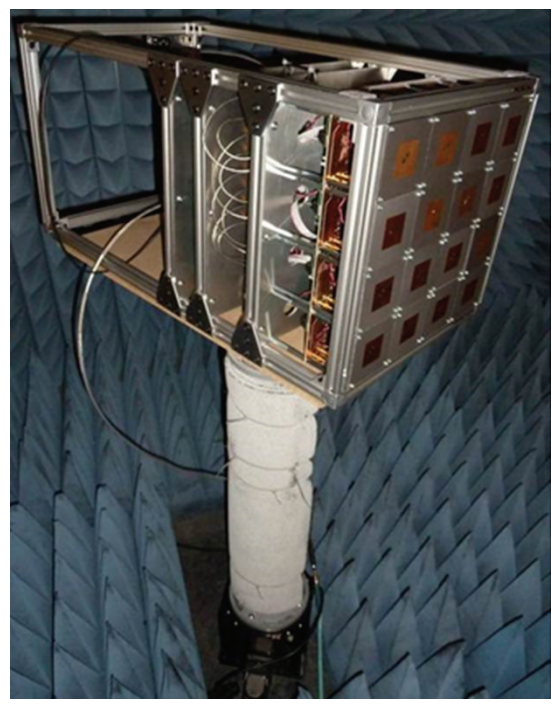

(a) Planar array

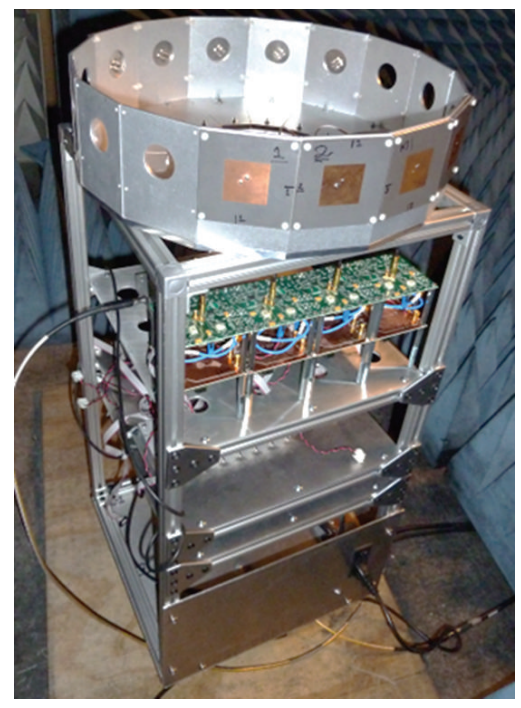

(b) Circular array

Figure 7: The CPAD is mounted on the rotary stage for antenna positioning.

(AZ) angle of the positioning system), as shown in Figures 7(a) and 7(b). The dual-polarized KOUN (KOUN is a prototype dual-pol. WSR-88D radar system) feed-horn was used as the source antenna, which was connected to the transmitting signal generating port of the PNA. The transmit power was $9 \mathrm{dBm}$. The PNA collects, via the coaxial cable, the received signals from the antenna array; it controls the positioning system simultaneously. The network analyzer is programmed to collect 200 samples of received signals between $2700 \mathrm{MHz}$ and $2710 \mathrm{MHz}$. The software invokes a script to increment a one-degree angle in $\mathrm{AZ}$ each time the script is executed. Then the VNA software collects the data from the test antenna and saves the data as a text file with a unique name. This simple setup can collect measurements over a 180-degree azimuth angle range for the CPAD with one-degree resolution. Measurements with antenna tapering are done by changing the attenuation of regions 2 and 3 of the antenna elements listed in Table 1 while maintaining zero attenuation in the elements of Region 1. The optimal amplitude tapering for achieving a minimum sidelobe level for the $4 \times 4$ array was computed using the particle swarm optimization method $[8,9]$, and the attenuation factors used in the measurements are shown in Figure 8.

\section{Planar Array Radiation Patterns}

The pattern function can be derived using Fourier series methods as in [10]. 


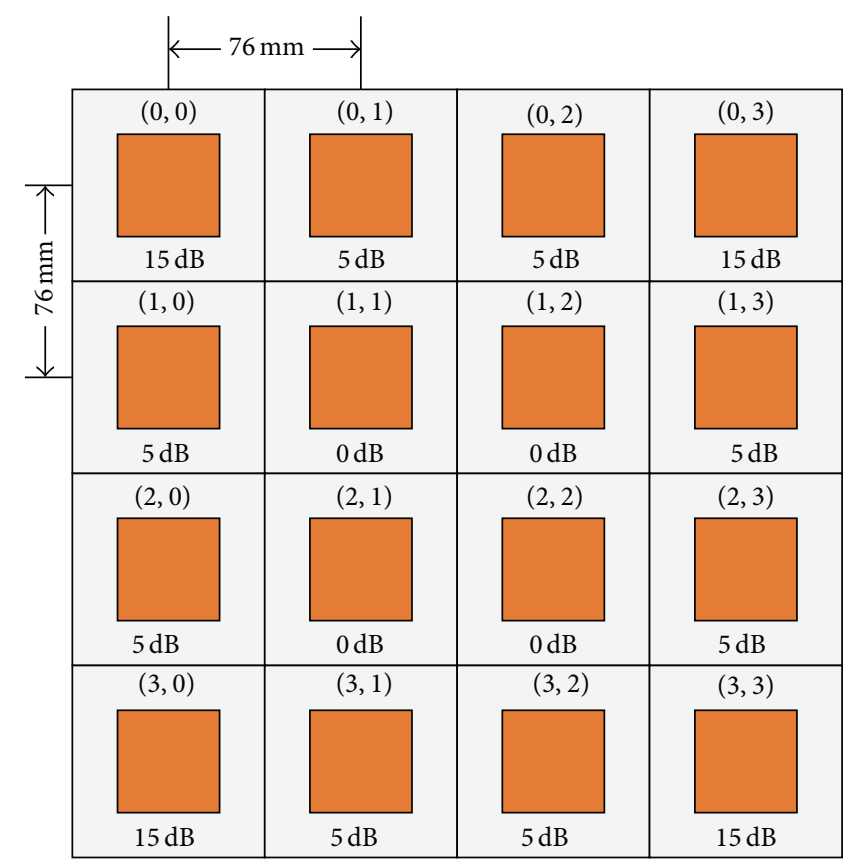

FIGURE 8: Attenuation map for each element for optimum tapering in theory.

5.1. Receive Array: Beam Steering without Amplitude Tapering. Figure 9 presents the measurement results of polarimetric planar antenna array patterns for different azimuth scanning angles. No amplitude tapering was applied. Measured copolarization (Co) and cross-polarization (X) patterns are based on vertical $(\mathrm{V})$ or horizontal $(\mathrm{H})$ polarizations of the source/transmit horn (according to the nature of the measurement method, definition 3 of cross-polarization [11] is used). The simulated co- and cross-polarization patterns are based on an open-source finite-difference time-domain (FDTD) EM solver [12]. A good agreement between the measurement and simulation radiation patterns of the copolarization and the power level of the cross-polarization patterns are observed for most of the steering angles.

Note that as the array scans electronically, the crosspolarization pattern also varies, and a cross-polarization peak appears in a similar direction as the main beam steers offbroadside. At broadside, the full-wave simulation pattern has a deep null while the measurement pattern results generally do not show this null. This is mainly due to the KOUN horn itself having a peak (about $-35 \mathrm{~dB}$ ) in the crosspolarization pattern. Considering that the antennas used in this configuration were simple probe-fed patched antennas, these initial results help general studies of planar array crosspolarization pattern evolution with different steering angles, while the power level of the cross-polarization pattern may be further suppressed if the radiating elements are replaced by more sophisticated designs.

5.2. Receive Array: Beam Steering with Optimal Amplitude Tapering. Amplitude tapering is a common technology to reduce the array pattern sidelobes (e.g., to below $-20 \mathrm{~dB}$ in this experiment) at the cost of a broader beamwidth. As mentioned in Section 4, Figure 8, an amplitude tapering technique based on a nonlinear optimization procedure was used in the demonstrative experiment. The measurement results and comparison with the simulations are again shown in Figure 10. Minor adjustments to the tapering factors were applied to the theoretical values during the measurements (Figure 11) to take account of the attenuator quantization effects. In the measurement results shown in Figure 10, the copolarized $\mathrm{V}$ and cross-polarized $\mathrm{H}$ patterns match the simulation results very well. The $H$ copolarized pattern has noticeable distortion at small positive steering angles, which is possibly due to some interference by the $\mathrm{H}$ polarization within the chamber setup.

\subsection{Transmit Array: Mirror Configuration for Reducing Cross-} Polarization Levels. The mirror array configuration [13] has been introduced in previous studies as a possible method for reducing cross-polarization levels. The CPAD enables researchers to configure the planar array with a different mirror manifold and different numbers of antenna elements. The pattern of mirror configuration is easy to obtain with transmit arrays. Figure 12 shows a simple $2 \times 2$ mirror configuration as an example. A comparison of the measured transmit array pattern for the mirror and nonmirror configurations is illustrated in Figure 13. As is seen, the mirror configuration indeed reduces the cross-polarization power levels. The most significant improvement, however, is around broadside. Measurements are also performed for the mirror configuration and array beam steering to off-broadside, and 


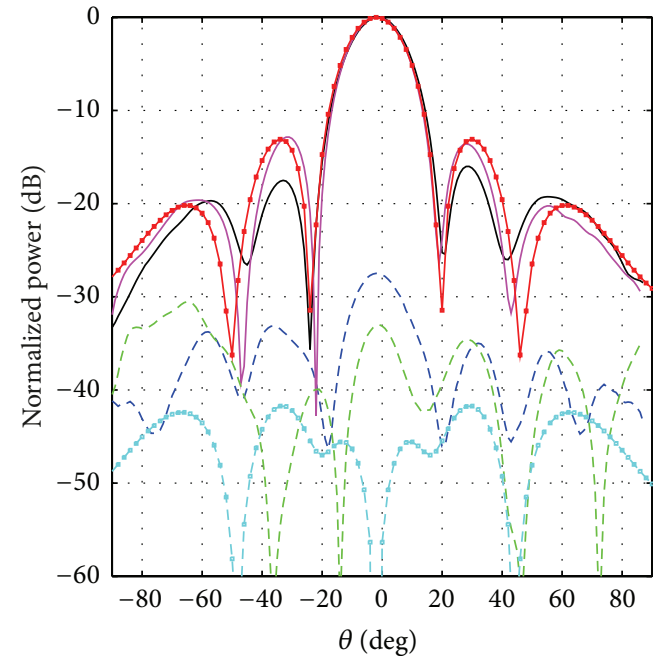

(a) $\phi=0^{\circ}$

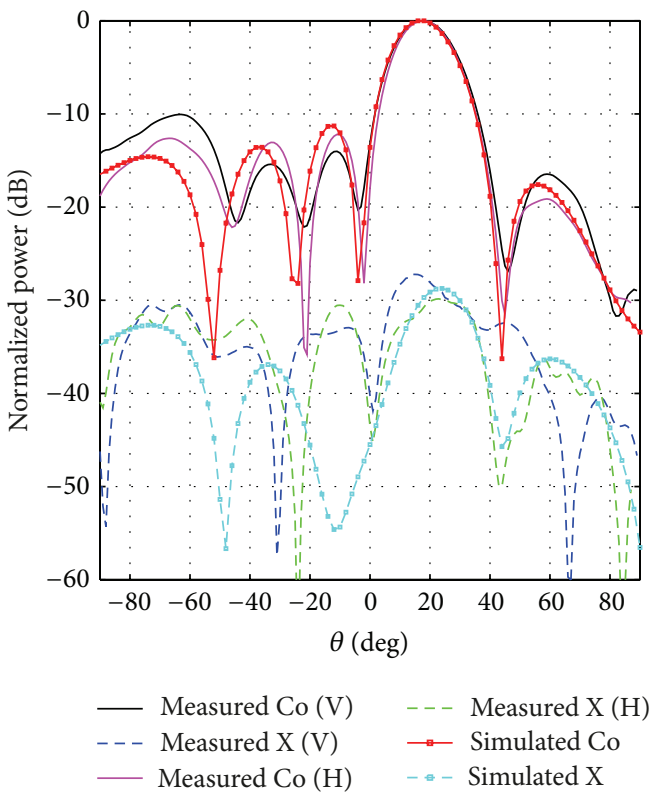

(c) $\phi=20^{\circ}$

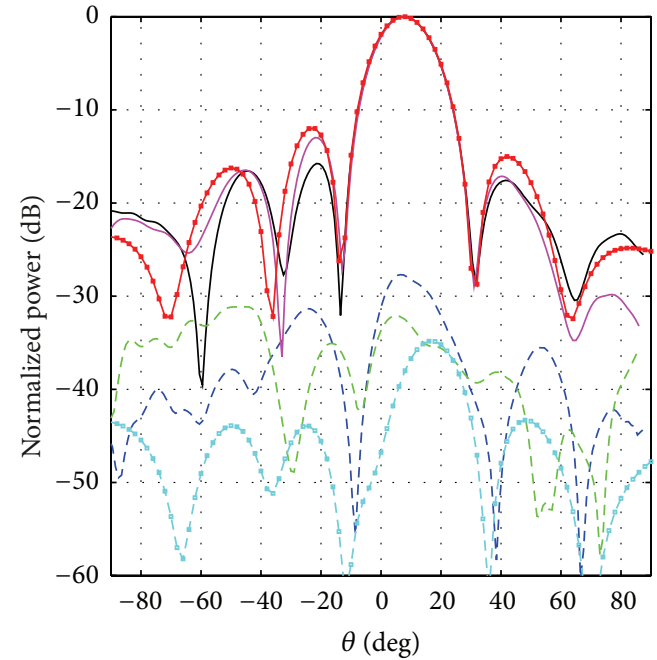

(b) $\phi=10^{\circ}$

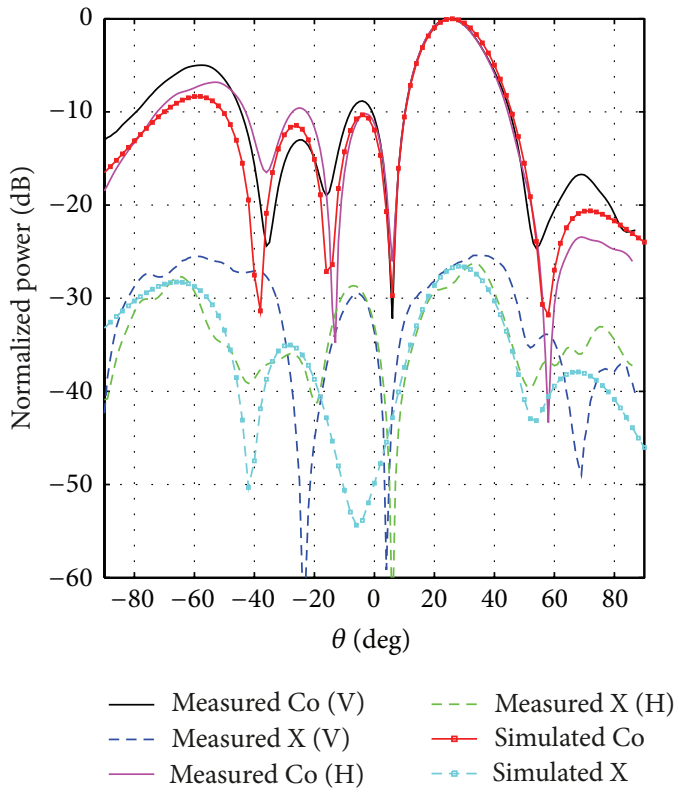

(d) $\phi=30^{\circ}$

FIGURE 9: Measured polarimetric $4 \times 4$ planar array patterns and comparison with simulations. Beam-steering angle $\phi$ is in azimuth direction. Simulations are performed using FDTD.

here the cross-polarization isolation performance degrades quickly, which indicates that a mirror configuration designed for broadside cross-polarization cancellation may not be appropriate for off-broadside arrays.

5.4. Transmit Array: 3D Amplitude and Phase Patterns. The transmit radiation pattern of the CPAD can be characterized using near-field measurement techniques (the low-cost nearfield measurement tool EMSCAN was used for the measurements). Figure 14 depicts the 3D amplitude and phase pattern measurement results. The basic profiles of these patterns match the corresponding simulation results. The 3D pattern (including phase) characterization is an important step in obtaining complete knowledge about polarimetric array radiations and how to achieve good calibrations. It is shown that the CPAD enables such measurements.

\section{Circular Array Radiation Patterns}

In this study, we provided electronic beam steering results for a circular array mainly to demonstrate the capability of the testbed and to investigate the fundamental properties of a conformal array manifold and currently not to compare patterns using electronic beam steering of the planar array 


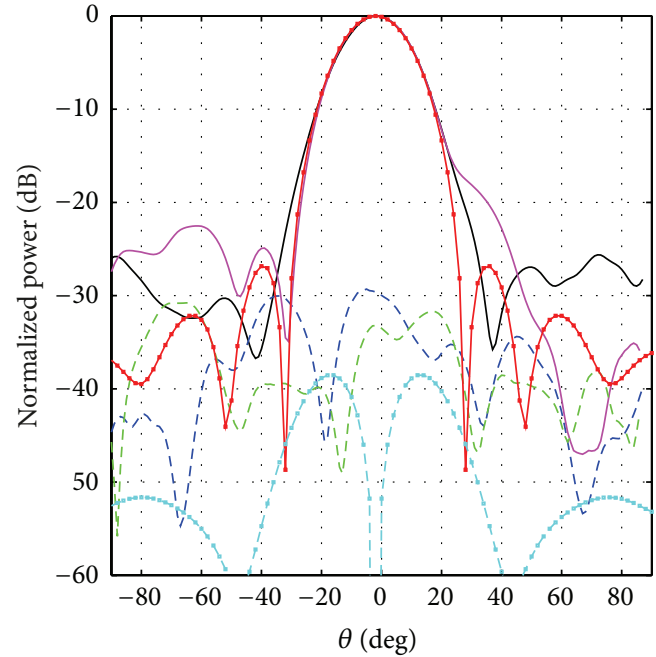

(a) $\phi=0^{\circ}$

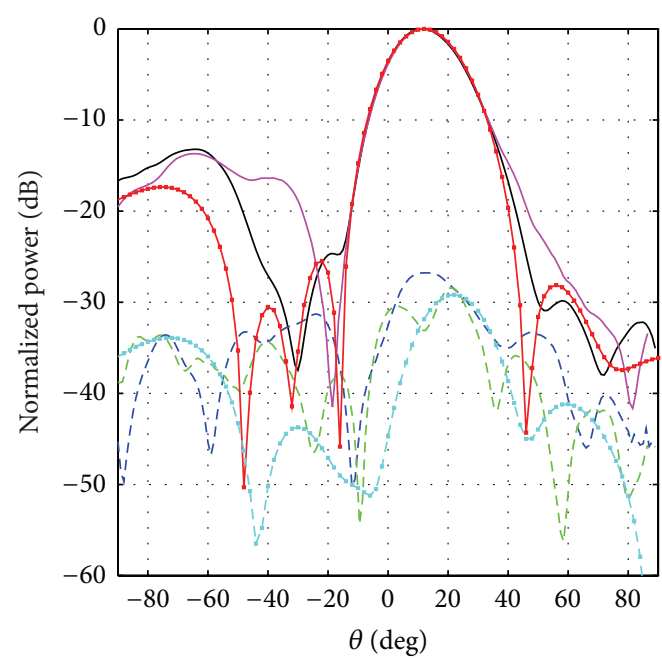

(c) $\phi=10^{\circ}$

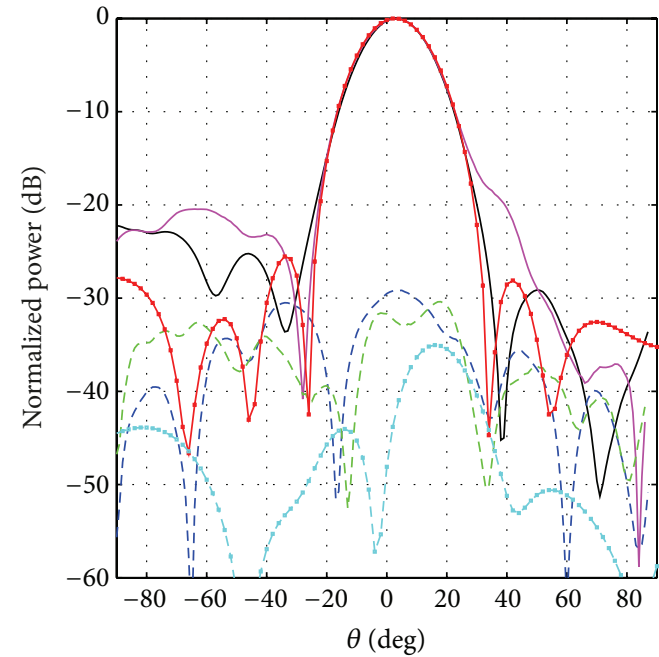

(b) $\phi=5^{\circ}$

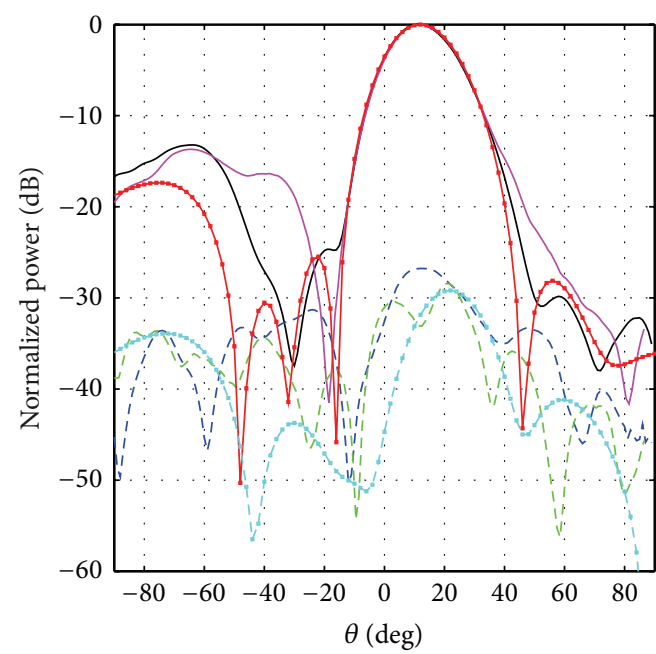

(d) $\phi=15^{\circ}$

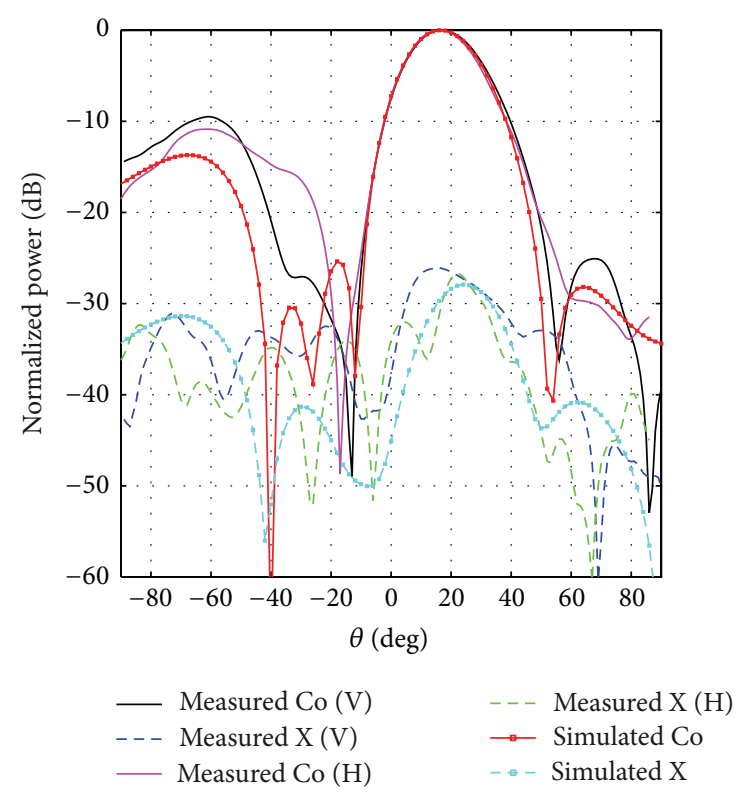

(e) $\phi=20^{\circ}$

FIGURE 10: Measured polarimetric array patterns with actual amplitude tapering. Beam-steering angle $\phi$ is in azimuth direction. Simulations are performed using FDTD. 


\begin{tabular}{|c|c|c|c|}
\hline 0.1887 & 0.551 & 0.551 & 0.1887 \\
\hline 0.551 & 1 & 1 & 0.551 \\
\hline 0.551 & 1 & 1 & 0.551 \\
\hline 0.1887 & 0.551 & 0.551 & 0.1887 \\
\hline
\end{tabular}

(a) Theoretical

\begin{tabular}{|c|c|c|c|}
\hline 0.1778 & 0.562 & 0.562 & 0.1778 \\
\hline 0.562 & 1 & 1 & 0.562 \\
\hline 0.562 & 1 & 1 & 0.562 \\
\hline 0.1778 & 0.562 & 0.562 & 0.1778 \\
\hline
\end{tabular}

(b) Actual

FIGURE 11: Theoretical and actual amplitude tapering values (linear scale values are displayed here in order to compare the configurations).

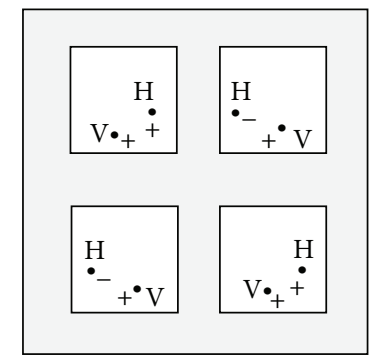

Figure 12: A simple $2 \times 2$ mirror array configuration of the CPAD.

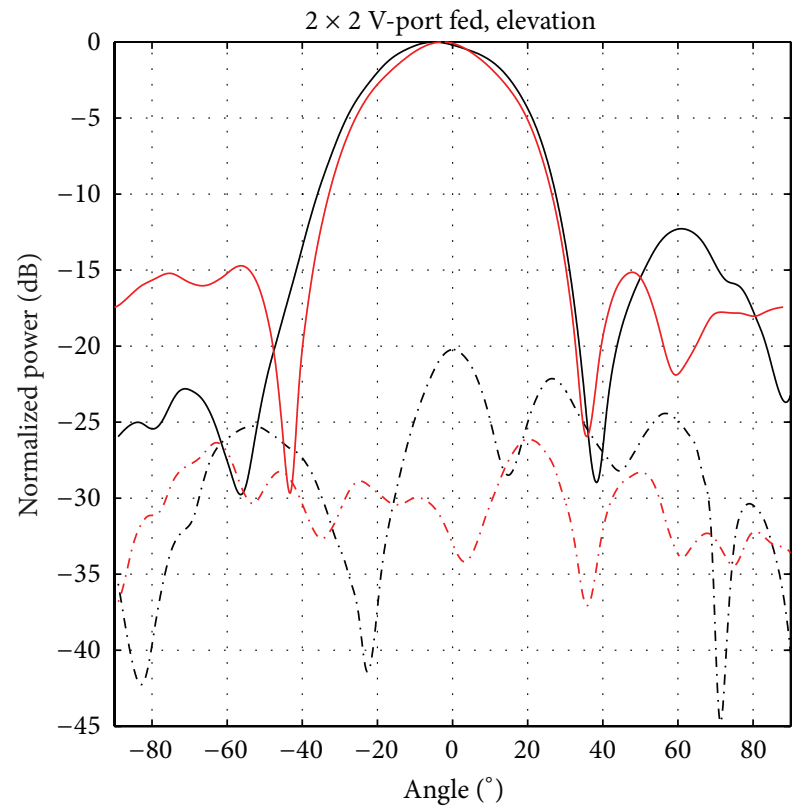

$\begin{array}{ll}\text { - Co-pol. nonmirror } & \text { Co-pol. mirror } \\ \text {-..- X-pol. nonmirror } & -.- \text { X-pol. mirror }\end{array}$

(a) Vertical polarization pattern

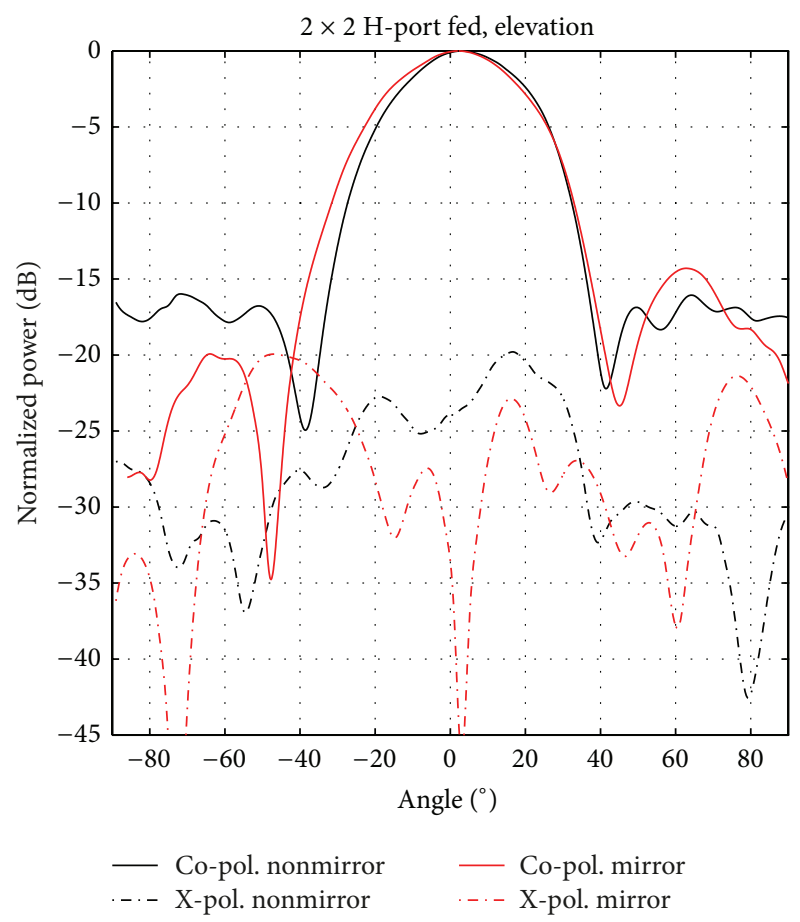

(b) Horizontal polarization pattern

FIGURE 13: Measured radiation patterns for the $2 \times 2$ arrays. 


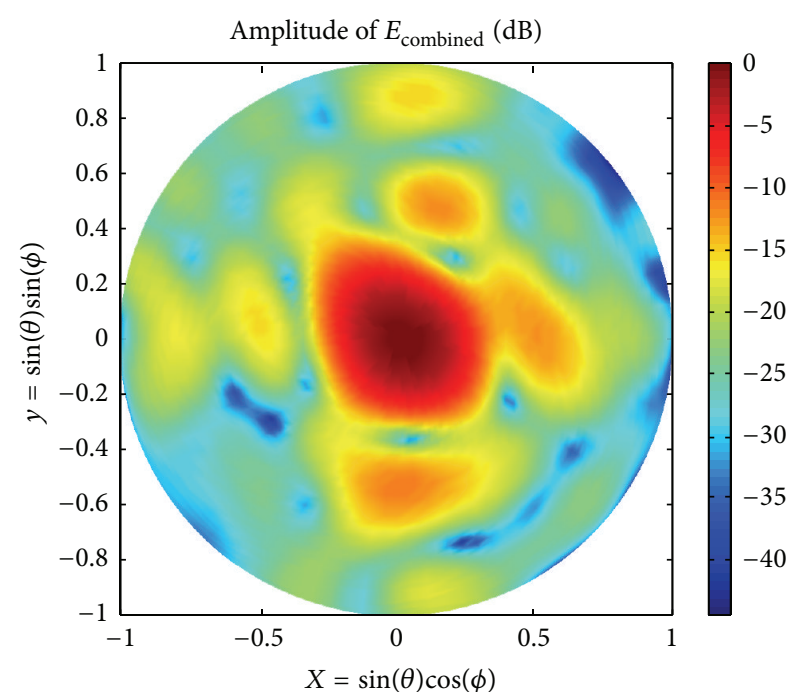

(a) $\left|E_{\theta}+E_{\phi}\right|$

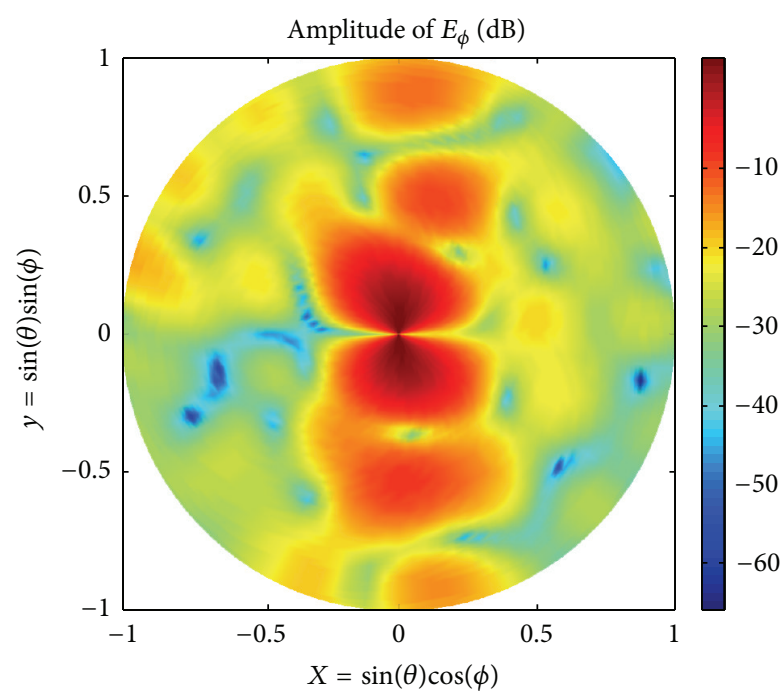

(c) $\left|E_{\phi}\right|$

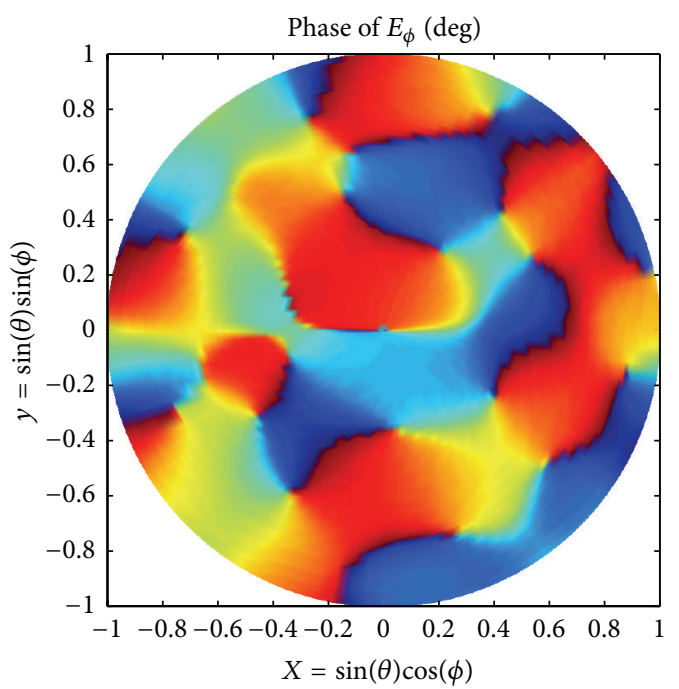

(e) $\angle E_{\phi}$

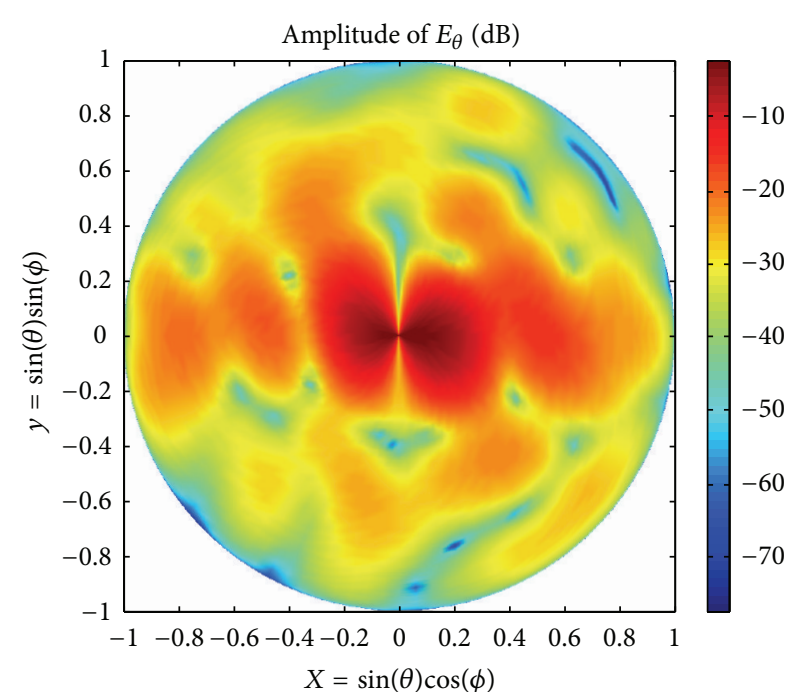

(b) $\left|E_{\theta}\right|$

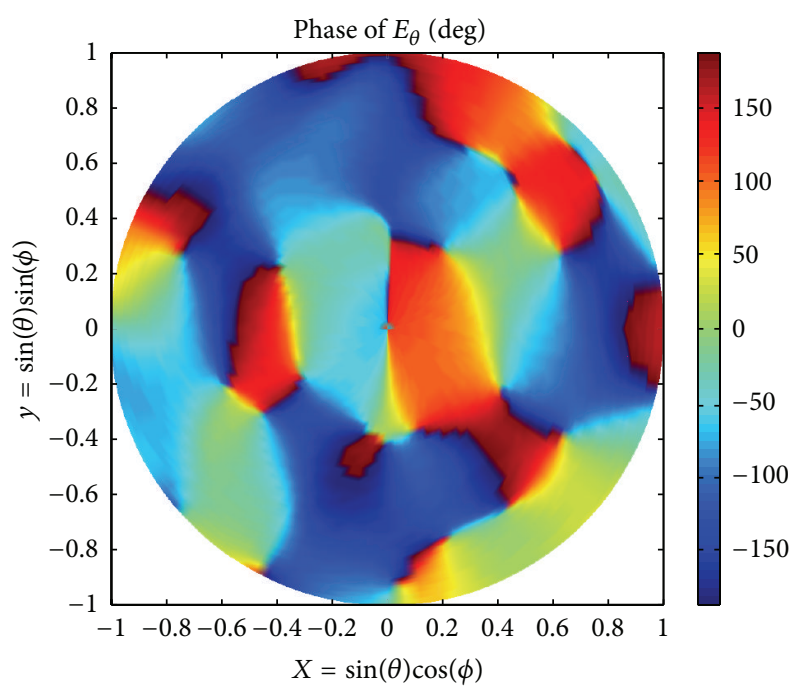

(d) $\angle E_{\theta}$

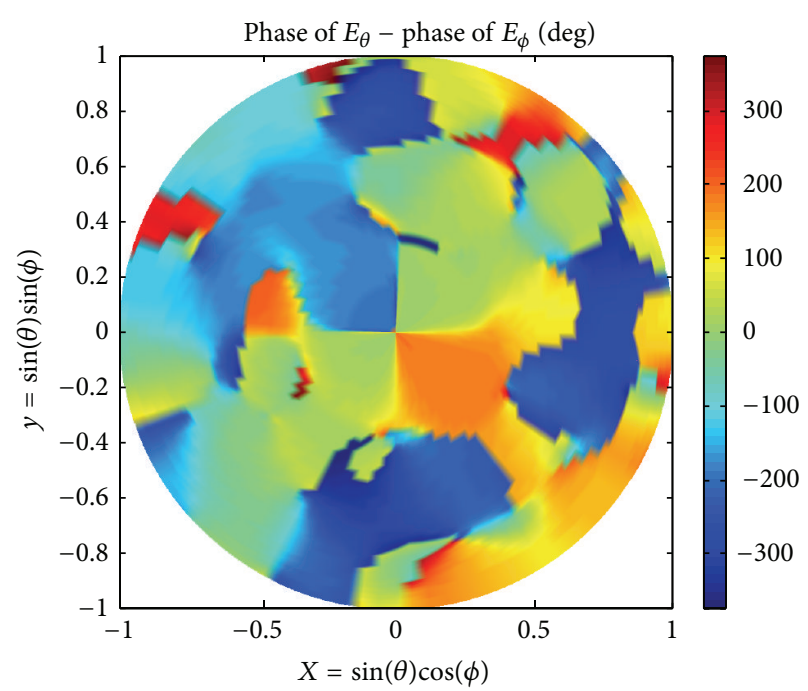

(f) $\angle E_{\theta}-\angle E_{\phi}$

FIGURE 14: Measured 3D transmitting pattern of the planar $4 \times 4$ (nonmirror) CPAD configuration. 


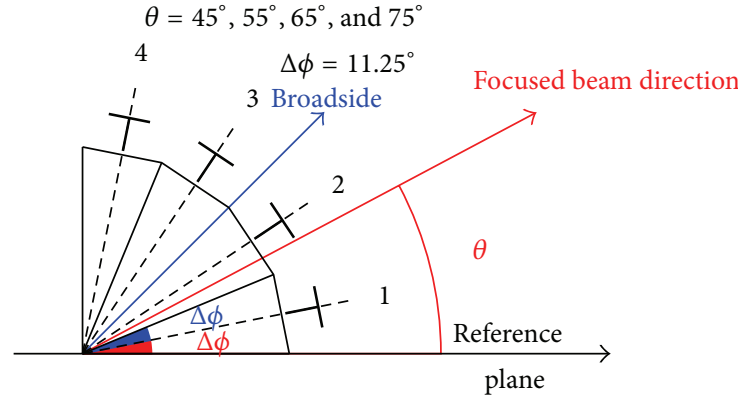

(a) Element arrangement

\begin{tabular}{ccccc}
\hline \multicolumn{5}{c}{$\theta-45^{\circ}$} \\
\hline Element & $0^{\circ}$ & $10^{\circ}$ & $20^{\circ}$ & $30^{\circ}$ \\
1 & -157 & -89 & -8 & 85 \\
2 & 110 & 141 & 189 & -107 \\
3 & 110 & 98 & 106 & 131 \\
4 & -157 & 151 & 116 & 100 \\
\hline
\end{tabular}

(b) Phase taper (in degrees) for a focused beam

FIGURE 15: Configuration of a 4-element circular array.

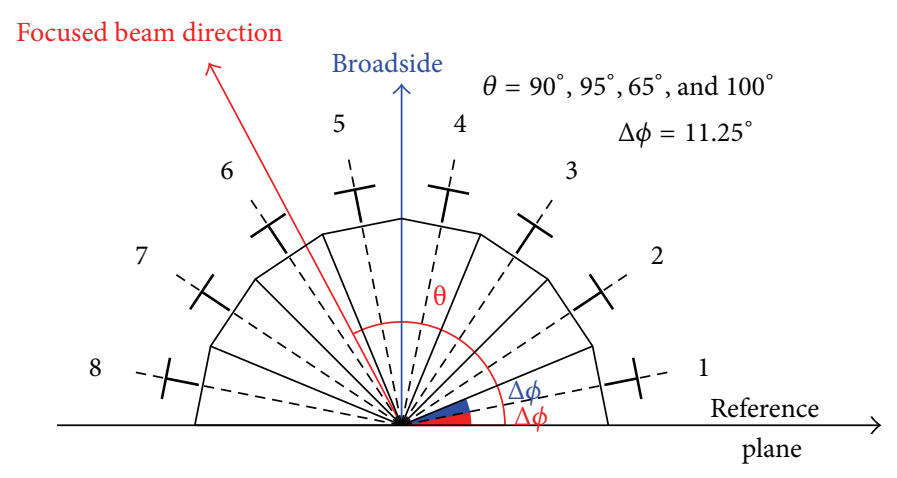

(a) Element arrangement

\begin{tabular}{cccc}
\hline \multicolumn{4}{c}{$\theta-90^{\circ}$} \\
\hline Element & $0^{\circ}$ & $5^{\circ}$ & $10^{\circ}$ \\
1 & -121 & -68 & -16 \\
2 & 15 & 61 & 107 \\
3 & -157 & -125 & -91 \\
4 & 110 & 123 & 140 \\
5 & 110 & 102 & 99 \\
6 & -157 & 175 & 152 \\
7 & 15 & -29 & -68 \\
8 & -121 & -174 & 137 \\
\hline
\end{tabular}

(b) Phase taper (in degrees) for a focused beam

Figure 16: Configuration of an 8-element circular array.

with that obtained using the commutative beam steering of the circular array.

The phase mode excitation [14] technique is used to form the focused beam for a circular array. The excitation of the $n$th element $f(n)$ can be expressed as follows:

$$
f(n)=\exp \{j k R \cos [\theta-(2 n-1) \Delta \phi]\} .
$$

Then the phase taper of the radiating elements can be calculated as follows:

$$
\psi(n)=-k R \cos [\theta-(2 n-1) \Delta \phi]
$$

In (1) and (2), $n$ is the reference number of the radiating element, $\psi(n)$ is the phase taper of the $n$th element, $k$ is the propagation constant, $R$ is the radius of the circular array, $\theta$ is the steering angle of the focused beam, and $\Delta \phi$ is the element projected angle at the center of the circular array. For the CPAD, the radius $R$ is $1.736 \lambda$ and $\lambda$ is $110 \mathrm{~mm}$, and Figures 15(a) and 16(a) show the geometries for the calculation of the phase taper values for 4-element and 8-element arrays, respectively. The horizontal axis in these figures is the reference plane for all the angles. For the 4-element circular array, Figure 15(b) lists the phase taper values calculated for $0^{\circ}, 10^{\circ}, 20^{\circ}$, and $30^{\circ}$ beam steering using (2). Figure 17 shows the simulated and measured radiation patterns. For this case, the measured radiation patterns have sidelobes lower than $-10 \mathrm{~dB}$ and a cross-polarization level of less than $-25 \mathrm{~dB}$, both of which are comparable to the patterns of a planar array with a similar aperture dimension.

For the 8-element circular array, Figure 16(b) lists the phase taper values calculated for $0^{\circ}, 5^{\circ}$, and $10^{\circ}$ beam steering using (2). The measured sidelobes in this case are lower than $-15 \mathrm{~dB}$, and the cross-polarization is lower than $-22 \mathrm{~dB}$, as shown in Figure 18.

\section{Conclusion}

The development of a large-scale phased array radar system such as the future MPAR will need a method for cost-effective risk mitigation. Simulations are not sufficient for determining the precise performance, and building large-scale arrays for 


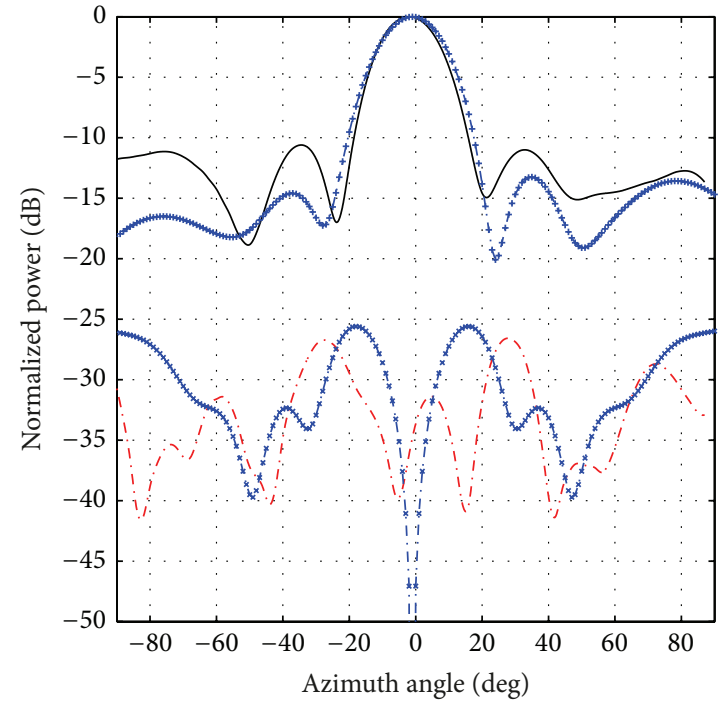

(a) $\theta_{b}=0^{\circ}$

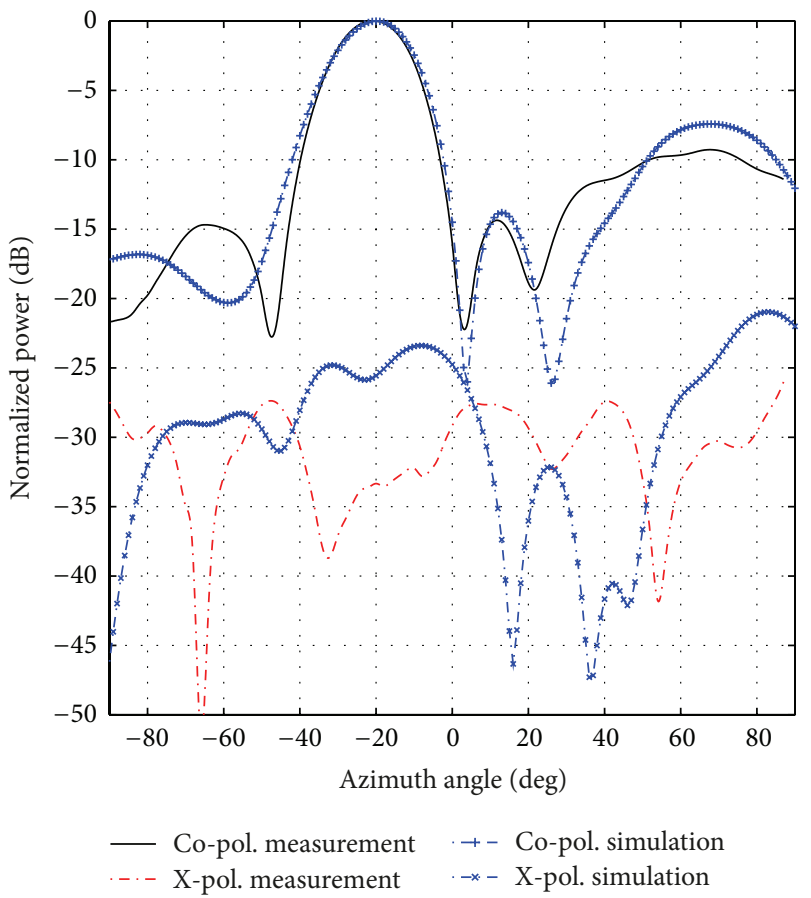

(c) $\theta_{b}=20^{\circ}$

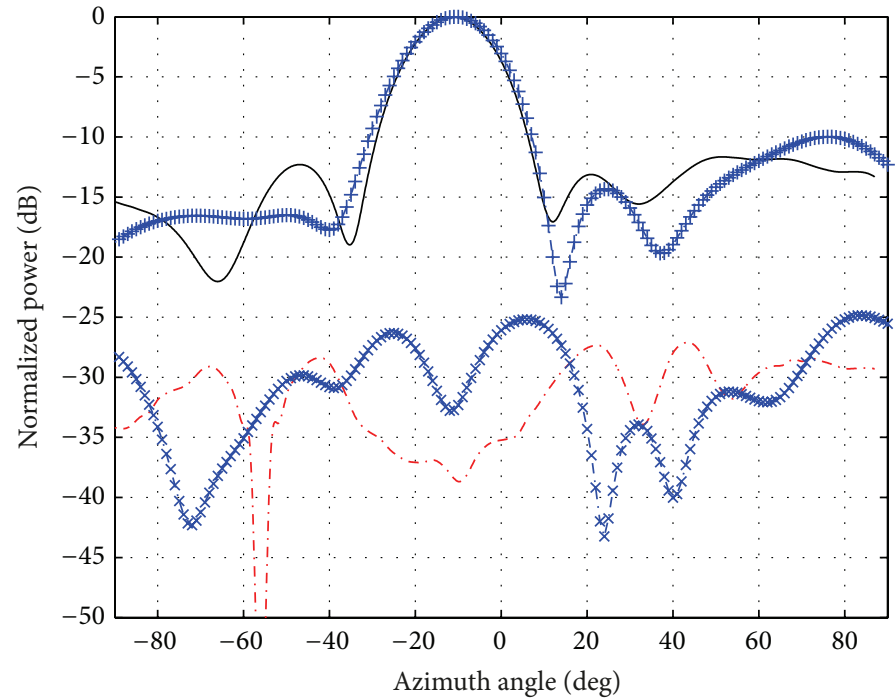

(b) $\theta_{b}=10^{\circ}$

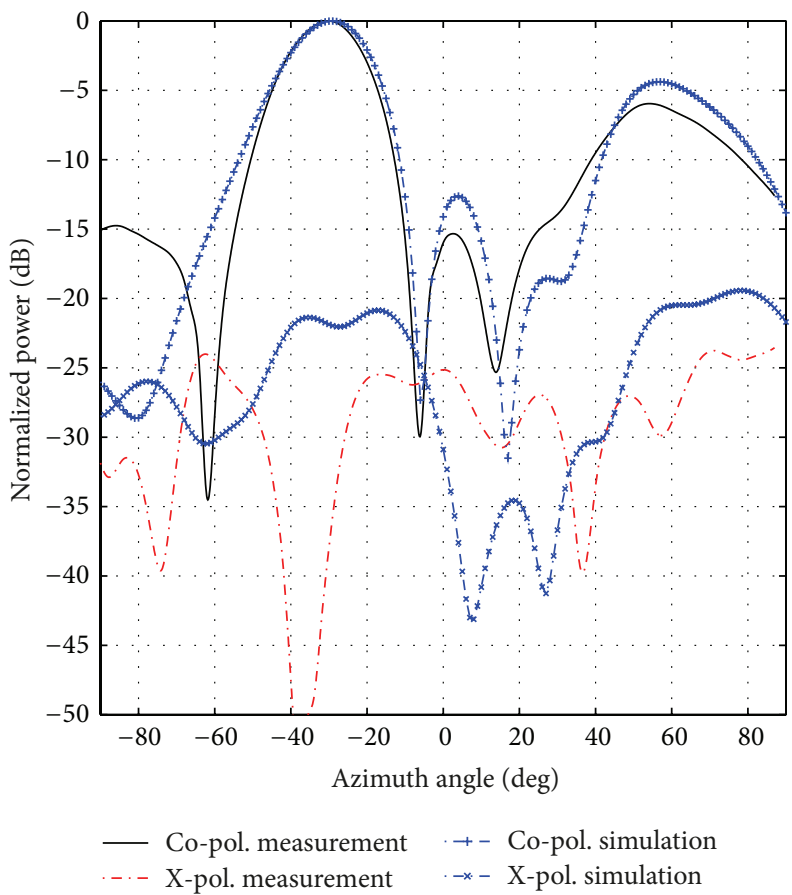

(d) $\theta_{b}=30^{\circ}$

FIGURE 17: Measured 4-element circular array patterns. Beam-steering angle $\theta_{b}$ is in azimuth direction. Simulations are performed using CST.

each new manifold idea is not realistic. Also, an educational tool is needed in the community to allow researchers to quickly acquire related knowledge. The CPAD provides a scalable, reconfigurable, and low-cost platform for testing and evaluating polarimetric phased array antennas and subarrays for radar systems. The comparative studies using simulation and measurements of different array manifolds demonstrate the effectiveness of the CPAD concept and its reconfiguration capabilities.

\section{Conflict of Interests}

The authors declare that there is no conflict of interests regarding the publication of this paper.

\section{Acknowledgment}

This work is supported by NOAA/NSSL through Grant no. NA080AR4320904. 


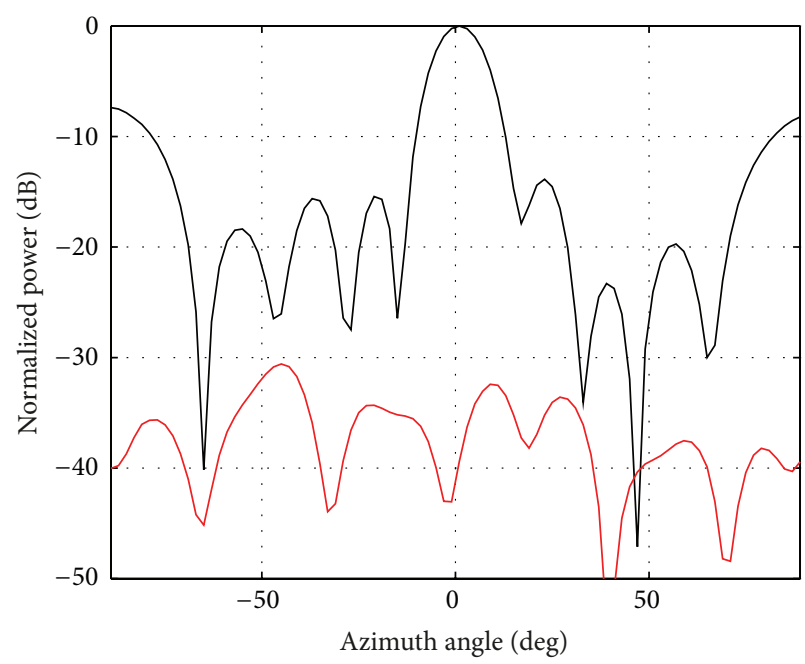

(a) $\theta_{b}=0^{\circ}$

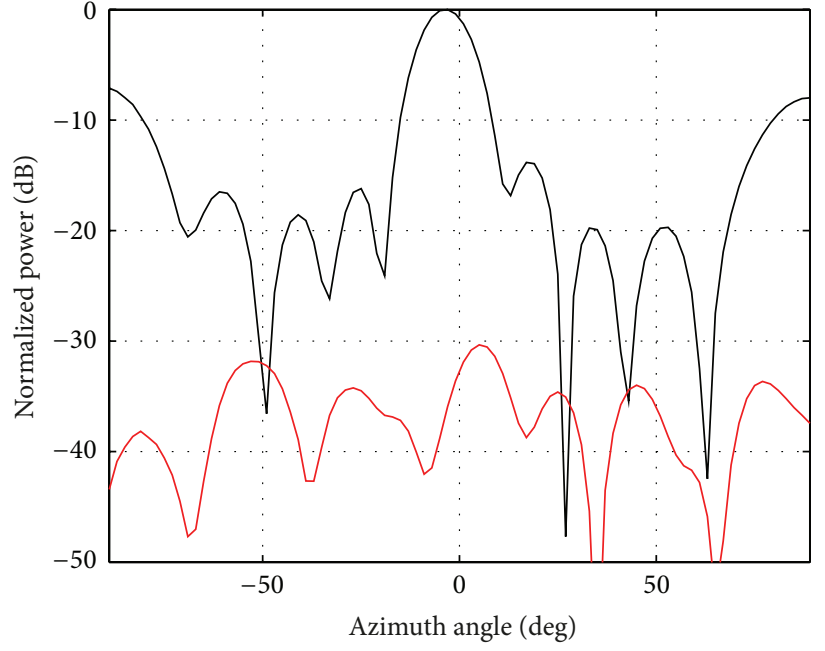

(b) $\theta_{b}=5^{\circ}$

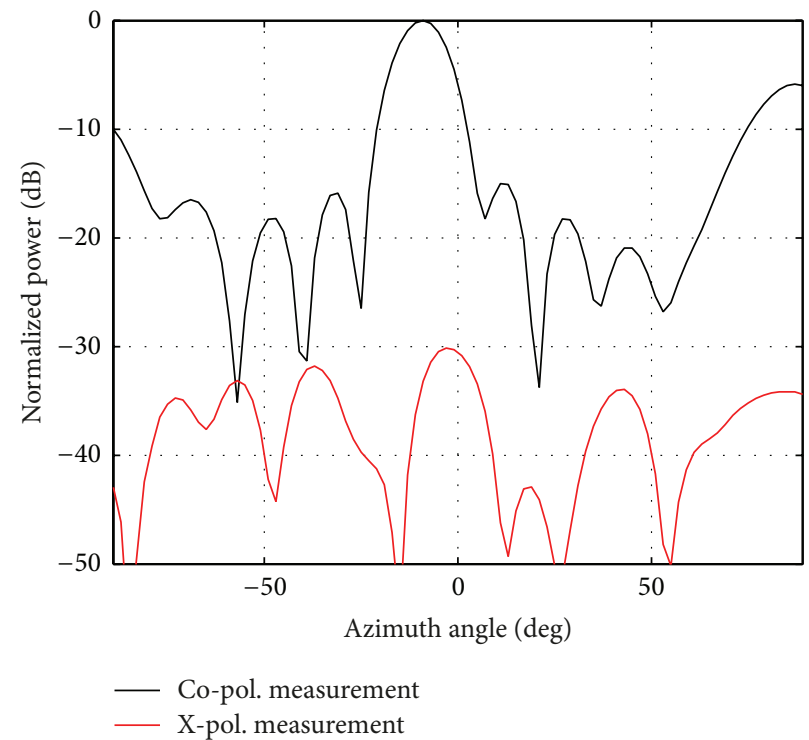

(c) $\theta_{b}=10^{\circ}$

Figure 18: Measured 8-element circular array patterns. Beam-steering angle $\theta_{b}$ is in azimuth direction.

\section{References}

[1] J. Herd, S. Duffy, M. Weber, G. Brigham, C. Weigand, and D. Cursio, "Advanced architecture for a low cost Multifunction Phased Array Radar," in Proceedings of the IEEE MTT-S International Microwave Symposium (MTT '10), pp. 676-679, usa, May 2010.

[2] MIT Lincoln Laboratory, "Multifunction phased array radar (MPAR) government proof of concept demonstrator," in Dual Polarization Patterns Extracted from Final Test Report Gen1 Panel Evaluation, MIT Lincoln Laboratory, Lexington, Mass, USA, 2012.

[3] G. Zhang, R. J. Doviak, D. S. Zrnić, R. Palmer, L. Lei, and Y. Al-Rashid, "Polarimetric phased-array radar for weather measurement: a planar or cylindrical configuration?” Journal of
Atmospheric and Oceanic Technology, vol. 28, no. 1, pp. 63-73, 2011.

[4] E. Brookner, "Mimo radar demystified," Microwave Journal, 2013.

[5] Y. Zhang, Y. Pan, S. Wang, Q. Zhao, D. Zrnic, and R. J. Doviak, "Reconfigurable and scalable array element design for polarimetric multifunctional PAR," in Proceedings of the IEEE Radar Conference: In the Eye of the Storm (Radar '11), pp. 175179, May 2011.

[6] Y. Rahmat-Samii, K. S. Kona, M. Manteghi et al., "A novel lightweight dual-frequency dual-polarized sixteen-element stacked patch microstrip array antenna for soil-moisture and seasurface-salinity missions," IEEE Antennas and Propagation Magazine, vol. 48, no. 6, pp. 33-46, 2006. 
[7] P. Gething, "High-frequency direction finding," Proceedings of the Institution of Electrical Engineers, vol. 113, no. 1, pp. 49-61, 1996.

[8] J. Robinson and Y. Rahmat-Samii, "Particle swarm optimization in electromagnetics," IEEE Transactions on Antennas and Propagation, vol. 52, no. 2, pp. 397-407, 2004.

[9] M. Benedetti, R. Azaro, D. Franceschini, and A. Massa, "PSObased real-time control of planar uniform circular arrays," IEEE Antennas and Wireless Propagation Letters, vol. 5, no. 1, pp. 545548, 2006.

[10] R. J. Mailloux, Phased Array Antenna Handbook, Artech House, Boston, Mass, USA, 2005.

[11] A. Ludwig, "The definition of cross polarization," IEEE Transactions on Antennas and Propagation, vol. 21, no. 1, pp. 116-119, 1973.

[12] T. Liebig, "openEMS—open electromagnetic field solver," General and Theoretical Electrical Engineering (ATE), University of Duisburg-Essen, http://openems.de/start/index.php.

[13] K. Woelders and J. Granholm, "Cross-polarization and sidelobe suppression in dual linear polarization antenna arrays," IEEE Transactions on Antennas and Propagation, vol. 45, no. 12, pp. 1727-1740, 1997.

[14] A. W. Rudge, The Handbook of Antenna Design, vol. 16 of IET, 1983. 

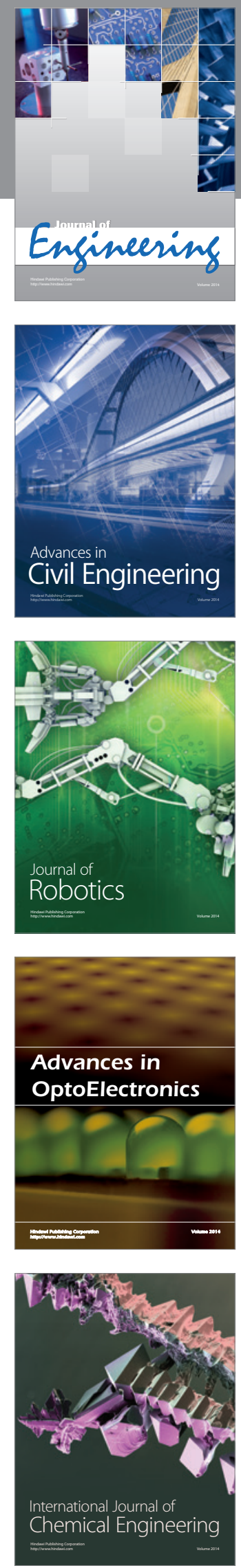

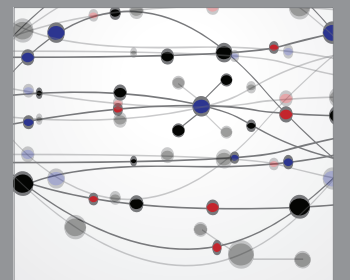

The Scientific World Journal
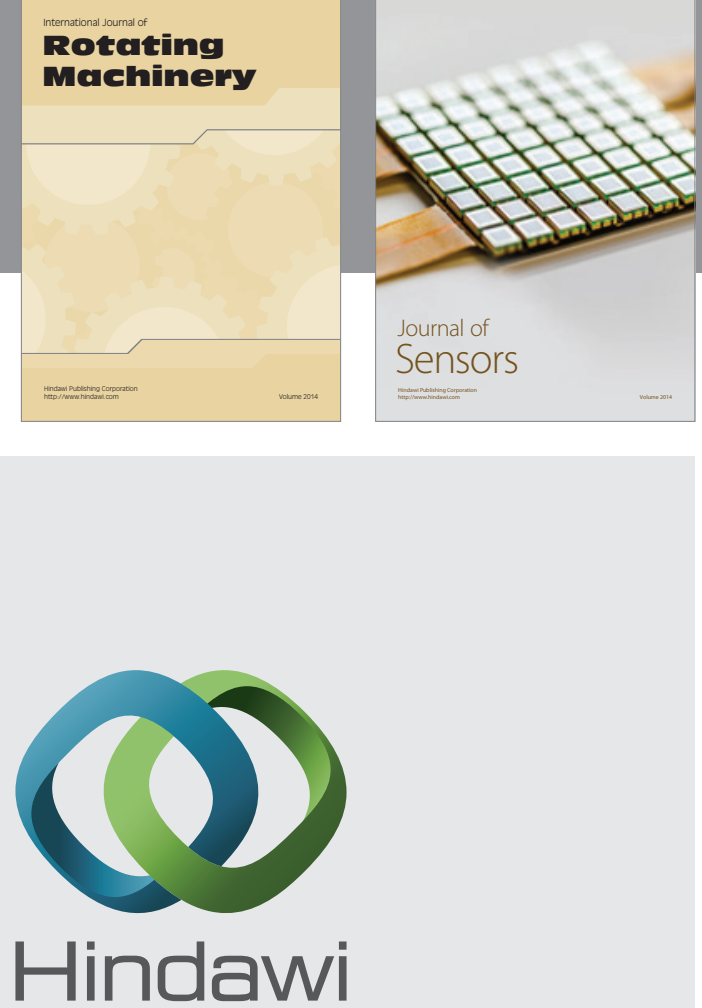

Submit your manuscripts at http://www.hindawi.com
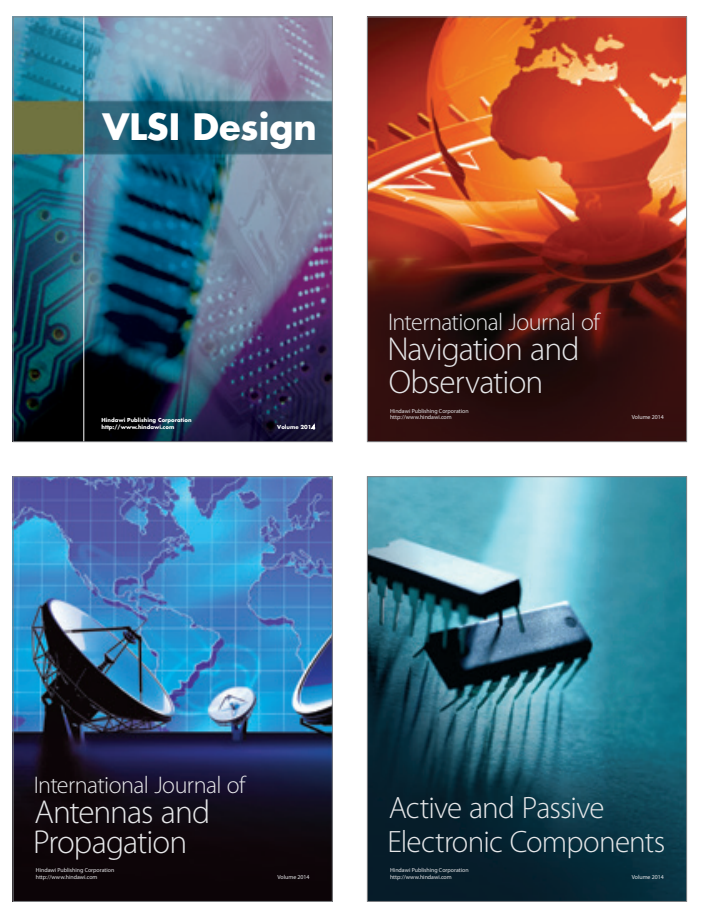
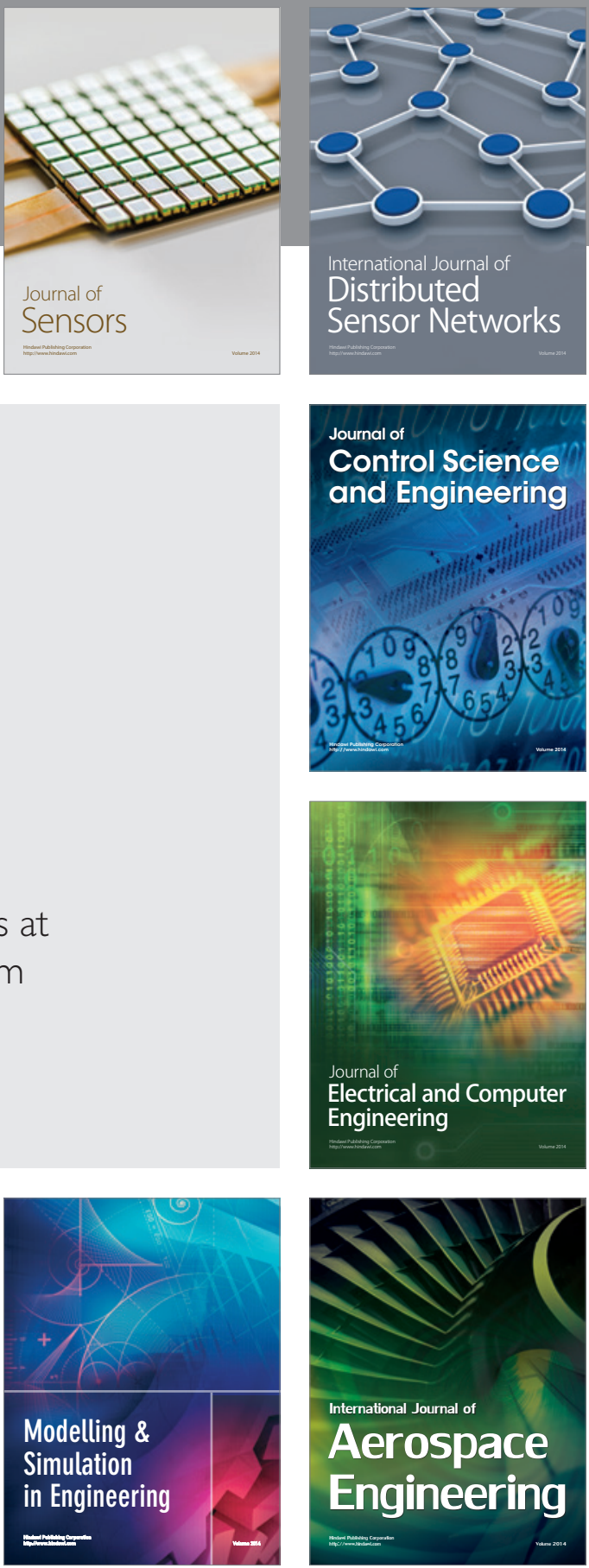

Journal of

Control Science

and Engineering
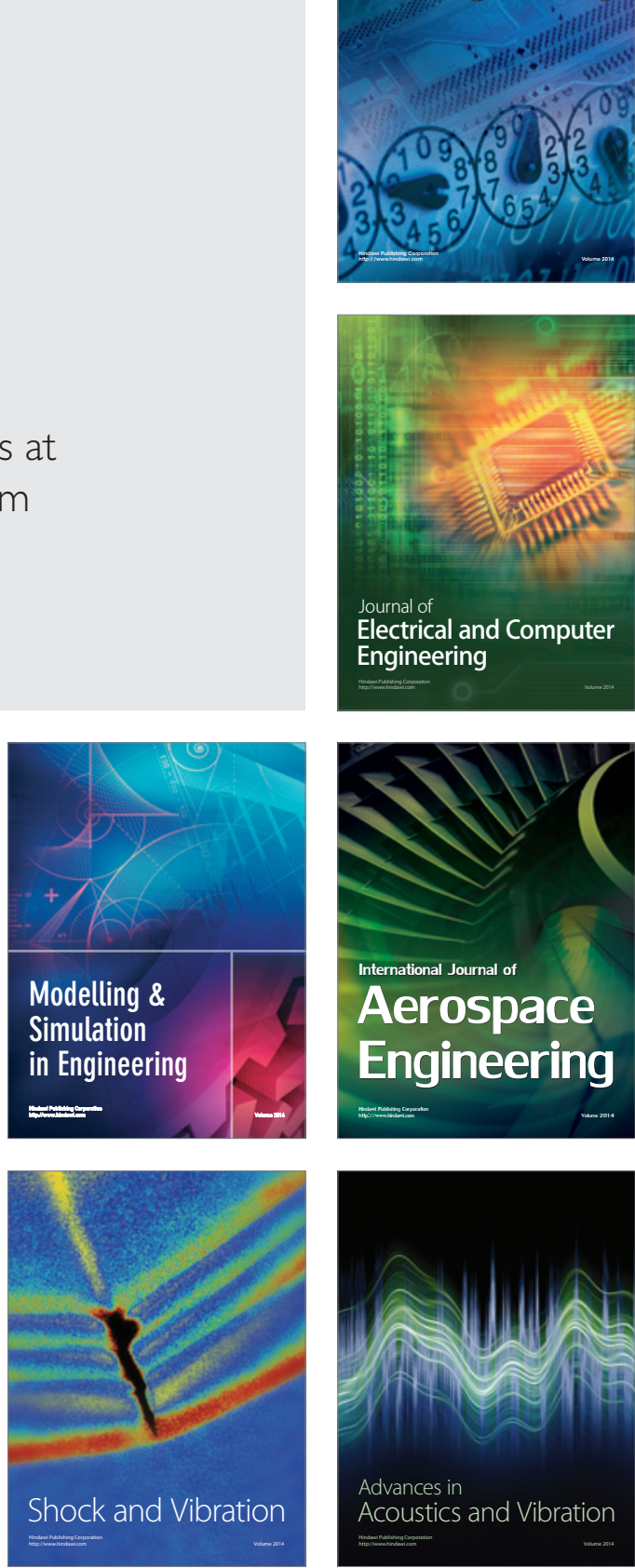